\title{
Supercontinuum generation in photonic crystal fibers for undergraduate laboratory
}

Gregory Helmininack, Derek Gladysiewski, Feng Zhou, Ken Hershman, Ben Campbell, et al.

Gregory Alan Helmininack, Derek D. Gladysiewski, Feng Zhou, Ken Hershman, Ben Campbell, Jeff Thomas, "Supercontinuum generation in photonic crystal fibers for undergraduate laboratory," Proc. SPIE 9665, Tenth International Topical Meeting on Education and Training in Optics and Photonics, 96651Y (3 June 2007); doi: 10.1117/12.2207809

SDIE Event: Tenth International Topical Meeting on Education and Training in Optics and Photonics, 2007, Ottawa, Ontario, Canada 


\title{
Supercontinuum Generation in Photonic Crystal Fibers for Undergraduate Laboratory
}

\author{
Gregory Alan Helmininack*, Derek D Gladysiewski*, Feng Zhou, and Ken Hershman \\ Department of Physics, Indiana University of Pennsylvania \\ 167 Northpointe Blvd, Freeport, PA 16201 USA \\ Ben Campbell and Jeff Thomas \\ Electro-Optics Center, Penn State University \\ 222 Northpointe Blvd, Freeport, PA 16229 USA
}

\begin{abstract}
The nanotechnology field is currently undergoing an exciting period of discoveries. It is necessary to bring nanotechnology to physics students. However, there is a lack of nanotechnology experiments developed for the undergraduate labs. By coupling high peak power laser pulses to a highly nonlinear photonic crystal fiber, supercontinuum generation and characterization are incorporated into nanotechnology education in undergraduate physics labs. Because of the fast advance and truly interdisciplinary nature of nanotechnology, the supercontinuum generation in photonic crystal fiber experiment gives physics undergraduate students an opportunity to work with high power lasers, to gain hands-on experience with state-of-art test and measurement equipment, and to access research projects in fiber optics, laser applications and nanotechnology.
\end{abstract}

\section{INTRODUCTION}

A supercontinuum is a broad spectrum beyond all visible colors with the properties of a laser. In other words, a supercontinuum is coherent white light. The first observation of a supercontinuum generated in a photonic crystal fiber (PCF) dates back to 1999 by Ranka et al. [1]. The progress in photonic crystal fibers [2] makes the supercontinuum generation relatively simple. As one of the most successful examples in nanophotonics, their applications have been reported ranging from sensors to lasers and to different types of passive and active waveguide devices.

The physics behind the process of supercontinuum generation in photonic crystal fibers has been studied. The dominant nonlinear effects responsible for the continuum generation are stimulated Raman scattering [3], four-wave mixing [4] and self-phase modulation [5], solitons [6], etc. The objective of this work is to generate and characterize supercontinuum in a highly nonlinear photonic crystal fiber pumped with femtosecond and picosecond laser pulses, and to study the effects of pump laser power, and pump wavelength on the supercontinuum generation.

\footnotetext{
${ }^{*}$ Undergraduate student.
}

Tenth International Topical Meeting on Education and Training in Optics and Photonics, edited by Marc Nantel, Proc. of SPIE Vol. 9665, 96651Y - (C) 2007 SPIE, OSA, IEEE, ICO doi: $10.1117 / 12.2207809$ 


\section{PHOTONIC CRYSTAL FIBERS}

Conventional optical fibers consist of a cladding and a core. The refractive index of a core is slightly higher than that of the cladding. Light propagates within the core of an optical fiber due to the total internal reflection. In recent years, rapid progress has been made in photonic crystal fibers and photonic crystal fiber devices. There are two basic types of photonic crystal fibers, namely, hollow core PCFs [7] and solid core PCFs. PCFs exhibit a novel cladding structure which is formed by a periodic pattern of air-holes around the central core. The air-holes lower the effective refractive index of the cladding. Hence, coupled light is guided within the core due to reflection from the core and cladding interface.

The photonic crystal fiber used in this work was from Thorlabs (Parts number: SC-5.01040). The fiber has a solid core of $4.8 \mu \mathrm{m}$ in diameter, surrounded by a periodic pattern of air-holes. Fig. 1 is a SEM image from the manufacturer that shows the central part of the fiber cross section. The outer diameter of the photonic crystal fiber is $125 \mu \mathrm{m}$. The PCF has a zero dispersion wavelength of $1040 \mathrm{~nm}$.

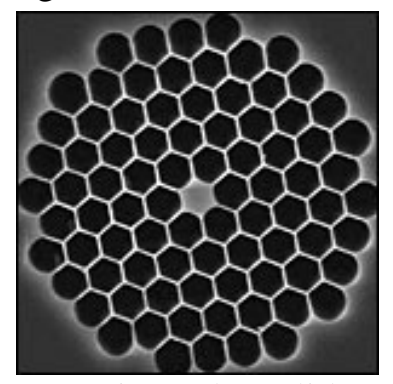

Fig. 1 SEM image of PCF cross section. The solid core is surrounded by a periodic pattern of air-holes (provided by Thorlabs).

\section{EXPERIMENT SETUP}

Both a femtosecond Ti:sapphire laser and a picosecond Nd:YAG laser were used as the source to generate the supercontinuum in the fiber. The energy per pulse $E_{\mathrm{p}}$ and peak power $P_{\mathrm{p}}$ from a mode-locked laser can be calculated by

$$
E_{p}=\frac{P_{a v}}{f_{r}} \text { and } P_{p}=\frac{P_{a v}}{T_{F W H M} f_{r}}
$$

where $P_{\mathrm{av}}$ is the average power from the laser; $f_{\mathrm{r}}$ is the laser repetition rate; $T_{\mathrm{FWHM}}$ is the full-width half-maximum (FWHM) of the laser pulses. For a $1 \mathrm{kHz}$ repetition rate, $200 \mathrm{fs}$ laser pulses at an average power of $20 \mathrm{~mW}$ from a Ti:sapphire laser, the calculated peak power is $100 \mathrm{MW}$. The energy per pulse is $20 \mu \mathrm{J}$. Similarly, for a $10 \mathrm{kHz}$ repetition rate, 10 ps laser pulses from a mode-locked Nd:YAG laser at $1.06 \mu \mathrm{m}$ with an average power of $20 \mathrm{~mW}$, the calculated peak power is $200 \mathrm{~kW}$ and energy per pulse is $2 \mu \mathrm{J}$.

The experimental setup of the supercontinuum generation in a PCF pumped by ultrashort pulses is illustrated in Fig.2. The TEM $\mathrm{T0}_{00}$-mode linearly polarized ultrashort pulses from a mode-locked laser first passed through a variable optical attenuator consisting of a halfwave plate, a polarization cube beamsplitter and a neutral density filter. Then the laser 
pulses were coupled into the PCF using a 20X objective lens having a numerical aperture of 0.4. The PCF used in the experiment had a length of 18 meters. The fiber ends were carefully stripped of its polymer coating, cleaned and cleaved to avoid any possible contamination. One end of the fiber was inserted into a fiber chuck which was mounted on a fiber coupler assembly with $\mathrm{x}, \mathrm{y}, \mathrm{z}$ and angle adjustments. The far end of the cleaved fiber was inserted into the bare fiber adapter for easy connection with the receptacles of a commercial optical power meter and an optical spectrum analyzer (OSA). The fiber coupler assembly with PCF was placed after the objective lens and aligned properly to obtain the best coupling of laser into the PCF.

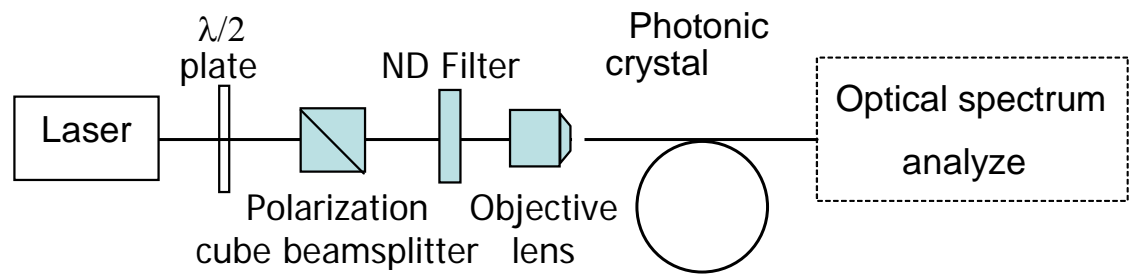

Fig. 2 Schematic drawing of the experimental setup for supercontinuum generation in a photonic crystal fiber pumped with ultrashort laser pulses.

Before the PCF was properly aligned, laser pulses with average powers of less than 1 $\mathrm{mW}$ were used to prevent any possible damage of the fiber end. Once the alignment and optimization were completed, the input power from a laser was gradually increased. The average power of the laser beam was measured using a Newport 1830-C Picowatt Digital Optical Power Meter with an 818-ST detector and an OD 3 calibrated optical attenuator (883-SL). The fiber coupling efficiency was measured to be approximately $30 \%$, although it could be further optimized by varying the objective lens magnification or the antireflection coatings. The spectrum from the PCF output was investigated with an optical spectrum analyzer (Ando AQ-6310B) which has a full spectrum range from 400 $\mathrm{nm}$ to $1750 \mathrm{~nm}$ with a resolution of $0.1 \mathrm{~nm}$.

\section{SUPERCONTINUUM GENERATION}

The interaction of ultrashort pulses with a nonlinear PCF leads to spectral broadening. The properties of the resulting supercontinuum depend primarily on the input pulse and PCF parameters. When the generated supercontinuum covers the whole visible spectral region, it appears as white light as shown in Fig. 3.
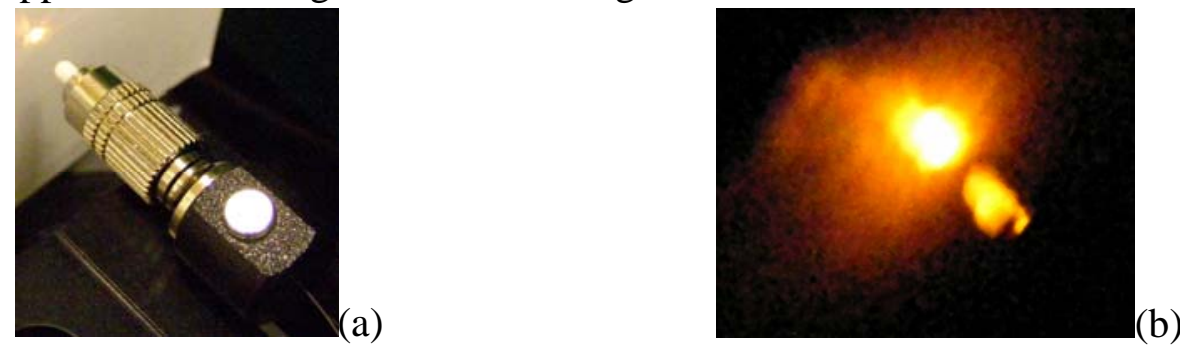

Fig.3 The supercontinuum shown on a screen; when the PCF was pumped with $775 \mathrm{~nm}$, $200 \mathrm{fs}$ pulses at (a) $10 \mathrm{~mW}$; and (b) $50 \mathrm{~mW}$. 
The evolution of the pump pulse spectrum into a supercontinuum in an 18-meter long photonic crystal fiber was studied by gradually increasing the pump pulse power. Figure 4 shows the measured spectra when the input laser power was $2 \mathrm{~mW}, 5 \mathrm{~mW}, 15 \mathrm{~mW}$ and $20 \mathrm{~mW}$, respectively, with the $\mathrm{x}$-axis being the wavelength and the $\mathrm{y}$-axis spectral density (a.u.) in log scale. Because the pump wavelength of $775 \mathrm{~nm}$ was shorter than the PCF zero dispersion wavelength of $1040 \mathrm{~nm}$, in the region of normal dispersion, the measured spectrum broadening was relatively narrow and most of the energy was concentrated around the pump wavelength. At higher pump power, the spectra were further broadened and the input energy converted to three visible bands around $580 \mathrm{~nm}, 660 \mathrm{~nm}$ and $750 \mathrm{~nm}$. The total spectral range was approximately over $500 \mathrm{~nm}$.

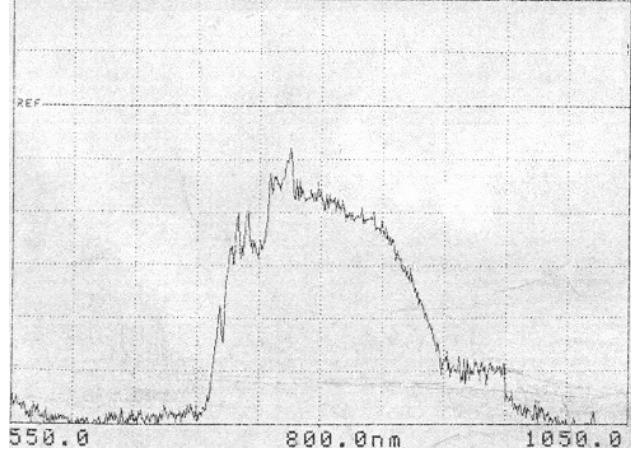

(a)

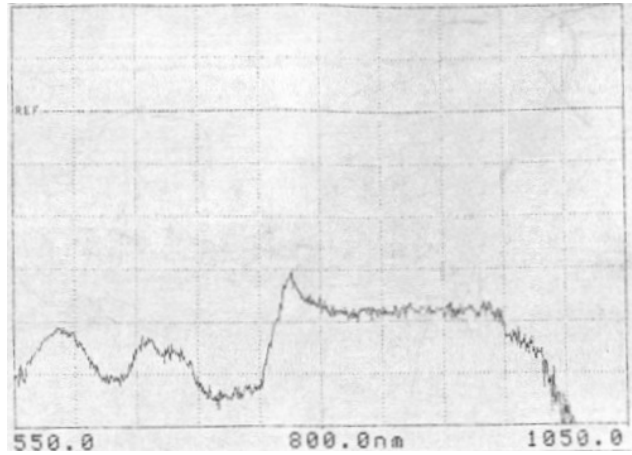

(c)

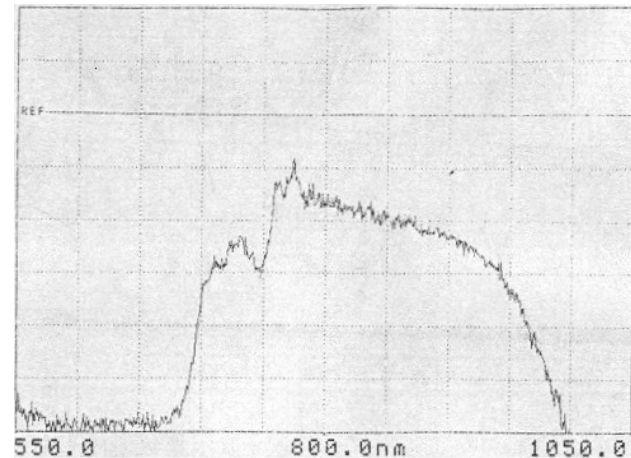

(b)

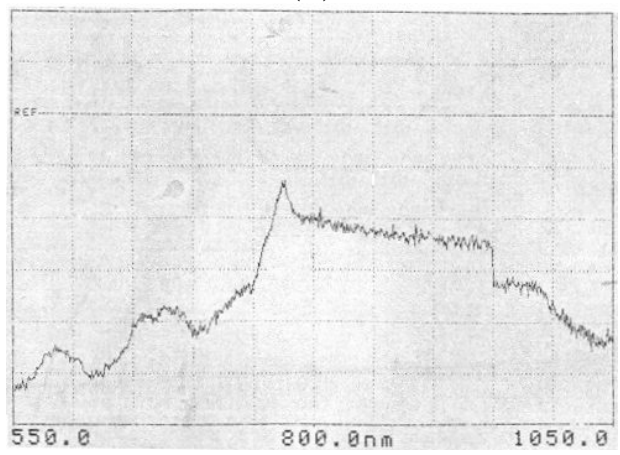

(d)

Fig. 5 The spectra evolution with the increase of input laser power: (a) $2 \mathrm{~mW}$; (b) 5 $\mathrm{mW}$; (c) $15 \mathrm{~mW}$; (d) $20 \mathrm{~mW}$. The $\mathrm{x}$-axis is the wavelength ranging from $550 \mathrm{~nm}$ to $1050 \mathrm{~nm}$, and the $y$-axis the spectral density (a.u.) in log scale.

The resulting supercontinuum was also observed and measured when the same fiber was pumped with 10 picosecond pulses at $1.06 \mu \mathrm{m}$ wavelength from a mode-locked Nd:YAG laser operated at a $10 \mathrm{kHz}$ repetition rate. Fig. 6 shows the measured spectra when pumped at $1 \mathrm{~mW}, 10 \mathrm{~mW}$ and $20 \mathrm{~mW}$. There was no supercontinuum observed when pumped at $1 \mathrm{~mW}$. At $15 \mathrm{~mW}$ input power, the spectrum was extended over the whole spectrum range limited by the instrument, but the initial laser input pulse was still visible. Since the PCF has a zero dispersion at $1.04 \mu \mathrm{m}$, the supercontinuum generated by 1.06 $\mu \mathrm{m}$ ultrashort laser pulses has a much broader spectrum range, as expected. At $20 \mathrm{~mW}$ input power, the PCF provides a relatively flat and very broad spectrum that covered the 
entire visible and near IR spectrum range from $400 \mathrm{~nm}$ to $1750 \mathrm{~nm}$ limited by the optical spectrum analyzer.
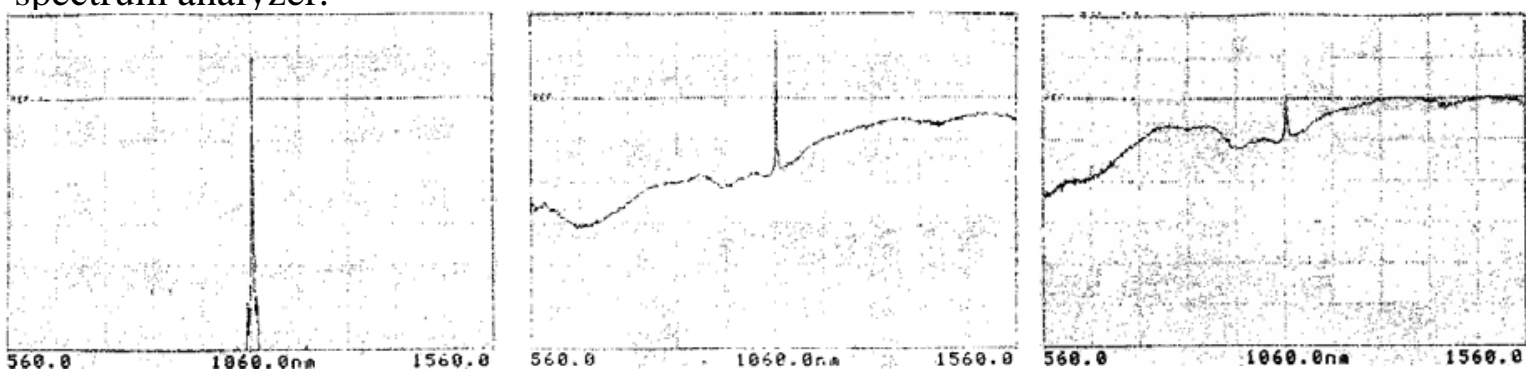

Fig. 6 The measured spectra pumped with $1.06 \mu \mathrm{m}, 10$ ps pluses at (a) $1 \mathrm{~mW}$; (b) $15 \mathrm{~mW}$ and (c) $20 \mathrm{~mW}$ average power. The $\mathrm{x}$-axis is the wavelength ranging from $560 \mathrm{~nm}$ to $1560 \mathrm{~nm}$, and the y-axis the spectral density (a.u.) in log scale.

\section{CONCLUSIONS}

In this work, supercontinuum generation from a photonic crystal fiber has been implemented using femtosecond and picosecond laser pulses for undergraduate physics labs such as fiber optics, lasers and spectroscopy. The use of an 18-meter long photonic crystal fiber made the supercontinuum generation possible at input power levels of only a few $\mathrm{mW}$. The evolution of the supercontinuum was investigated and the effects of pump power on the generated supercontinuum were measured with an optical spectrum analyzer. Because of the zero dispersion at $1040 \mathrm{~nm}$ wavelength, the input laser pulses at $1.06 \mu \mathrm{m}$ wavelength created a flat and broad supercontinuum over $1000 \mathrm{~nm}$ wide, limited only by the instrument, ranging from the entire visible spectrum to the near-IR.

\section{REFERENCES}

[1] J. K. Ranka, R. S. Windeler, and A. J. Stentz, "Visible continuum generation in airsilica microstructure optical fibers with anomalous dispersion at $800 \mathrm{~nm}$ ", Opt. Lett., Vol. 25, No. 1, pp. 25-27, 2000.

[2] P. St. J. Russell, "Photonic Crystal Fibers," Science, Vol 299, pp. 358-362, 2003

[3] S. Coen, A. Chau, R. Leonhardt, J. Harvey, J. Knight, W. Wadsworth, and P. Russell, "Supercontinuum generation by stimulated Raman scattering and parametric four-wave mixing in photonic crystal fibers," J. Opt. Soc. Am. B, Vol 19, pp. 753-764, 2002.

[4] W. J. Wadsworth, N. Joly, J. C. Knight, T. A. Birks, F. Biancalana, and P. St. J. Russell. Supercontinuum and four-wave mixing with Q-switched pulses in endlessly singlemode photonic crystal fibres. Optics Express, Vol 12(2), pp. 299-309, January 2004.

[5] Z. Zhu and T. G. Brown; "Effect of frequency chirping on supercontinuum generation in photonic crystal fibers," Optics Express, Vol 12(4), pp. 689-694, February 2004.

[6] K. Sakamaki, M. Nakao, M. Naganuma, and M. Izutsu; "Soliton induced supercontinuum generation in photonic crystal fiber," IEEE Journal of Selected Topics in Quantum Electronics, Vol 10(5), pp. 876-884, September 2004.

[7] R. F. Cregan, B. J. Mangn, J. C. Knight, T. A. Birks, P. Russell, P. J. Roberts, and D. C. Allan, "Single mode photonic and gap guidance of light in air," Science, Vol 285, pp. 1537-42, 1999. 